\title{
ON EXPONENTIALLY SEPARATED DIFFERENTIAL SYSTEMS
}

\author{
TAMASHA ALDIBEKOV
}

\begin{abstract}
Exponentially separated linear homogeneous system of ordinary differential equations with continuous limited coefficients in critical cases of Lyapunov exponents is considered. The generalized exponentially separated linear system of differential equations with regard to a monotonically increasing function is defined. It is established that if a linear homogeneous system of differential equations is generalized exponentially separated, Lyapunov's generalized exponents are stable in a class of small perturbations.
\end{abstract}

The work of Perron [12], see also [11, p. 193, theorem 9] was a source of definition of an exponential separation. Then these systems were studied in B.F.Bylov's [6], R.E. Vinograd's [7], V.M. Millionshchikov's [9], [10], Lillo's [8] works. The definition of an exponential separation has connection with the definition of an exponential dichotomy D.V. Anosov [5]. Some information on the theory of Lyapunov's generalized exponents is contained in Aldibekov T.M.'s works [1,2,3,4]. In the paper [3] the definition of an exponential separation is spread to linear systems with unlimited coefficients. In the present work using Lyapunov's generalized exponents, the subclass of linear systems with continuous limited coefficients is investigated, where the definition of an exponential separated loses its meaning.

The class of linear homogeneous systems of differential equations is considered

$$
\dot{x}=A(t) x
$$

where $t \in I \equiv\left[t_{0},+\infty\right),\left(t_{0}>1\right), x \in R^{n}, A(t)$ is a continuous matrix of the dimension $n \times n$, satisfying to the inequality

$$
\|A(t)\| \leq K \psi(t), \quad(K>0)
$$

$\psi(t)$ is a continuous, positive, distinct from a constant, such a fixed function that the function

$$
q(t)=\int_{t_{0}}^{t} \psi(\tau) d \tau, \quad q(t) \uparrow+\infty \text { as } t \uparrow+\infty
$$

2000 Mathematics Subject Classification. Primary 05C38, 15A15; Secondary 05A15, 15A18. 
and satisfies to the conditions:

$$
\varlimsup_{t \rightarrow+\infty} \frac{\ln t}{q(t)}=0, \varlimsup_{t \rightarrow+\infty} \frac{q(t)}{t}=0, \ln t<q(t)<t, \text { at } t \geq t_{0}>t .
$$

Note that Lyapunov's exponents of the linear system (0.1) accept zero values, i.e. a so-called critical case takes place.

The generalized exponent of a nonzero solution $x(t)$ of the linear system $(0.1)$ is determined by the formula

$$
\lambda[x, q] \equiv \varlimsup_{t \rightarrow+\infty} \frac{1}{q(t)} \ln \|x(t)\|
$$

The generalized exponents of the fundamental system of solutions, in which the sum of the generalized exponents of solutions is the smallest compared with other fundamental systems of solutions, are called Lyapunov's generalized exponents of the linear system (0.1).

As a rule, Lyapunov's generalized exponents of the linear system (0.1) are designated as follows

$$
\lambda_{n}(A) \leq \lambda_{n-1}(A) \leq \ldots \leq \lambda_{1}(A)
$$

Definition 0.1. The linear system (0.1) satisfying to the condition $(0.2)$ is called generalized exponentially separated, if it has solutions $x_{1}(t), \ldots, x_{n}(t)$, such that for all $t \geq s \geq t_{0}$ the inequalities are fulfilled

$$
\frac{\left\|x_{i-1}(t)\right\|}{\left\|x_{i-1}(s)\right\|} \div \frac{\left\|x_{i}(t)\right\|}{\left\|x_{i}(s)\right\|} \geq B e^{\alpha[q(t)-q(s)]}, \quad i=\overline{2, n}
$$

with some constants $\alpha>0, B \geq 1, q(t)=\int_{t_{0}}^{t} \psi(\tau) d \tau$.

Definition 0.2. If the linearly perturbed system

$$
\dot{x}=(A(t)+P(t)) x
$$

where a continuous matrix of perturbation $P(t), t \geq t_{0}$ satisfies to the conditions

$$
\|P(t)\| \leq K \psi(t) \text { at } t \geq t_{0} \text { and } \lim _{t \rightarrow \infty} \frac{\|P(t)\|}{\psi(t)}=0,
$$

has Lyapunov's generalized exponents, which coincide with generalized Lyapunov's exponents of the linear system (0.1), we can say that the linear system (0.1) satisfying to the condition (0.2) has Lyapunov's stable generalized exponents.

Theorem 0.3. A linear homogeneous diagonal system

$$
\frac{d x}{d t}=A_{d}(t) x
$$

where

$$
A_{d}(t)=\operatorname{diag}\left\{a_{1}(t), \ldots, a_{n}(t)\right\},\left\|A_{d}(t)\right\| \leq K \psi(t), \quad t \in I
$$


$a_{i}(t), i=1, \ldots, n$; are continuous functions satisfying to inequalities:

$$
a_{i-1}(t)-a_{i}(t) \geq \alpha \psi(t), \quad \alpha>0, \quad t \in I, \quad i \in\{2, \ldots, n\} ;
$$

has Lyapunov's stable generalized exponents.

Proof. Note the perturbed system

$$
\frac{d x}{d t}=\left(A_{d}(t)+P(t)\right) x
$$

in the coordinate form

$$
\frac{d x_{i}}{d t}=a_{i}(t) x_{i}+\sum_{k=1}^{n} p_{i k}(t) x_{k}, \quad i \in\{1, \ldots, n\}
$$

It is known that from $\lim _{t \rightarrow \infty} \frac{\|P(t)\|}{\psi(t)}=0$ it follows that for any $i \in\{1, \ldots, n\}$, $k \in\{1, \ldots, n\}$ the equality takes place

$$
\lim _{t \rightarrow \infty} \frac{\left|p_{i k}(t)\right|}{\psi(t)}=0
$$

Following the work [3, pp. $65-78]$ it is easily established that the linearly perturbed system (0.6) satisfying to the condition (0.7) has $n$ linearly independent solutions

$x_{k}=\left\{x_{1 k}, x_{2 k}, \ldots, x_{n k}\right\}, k=1,2, \ldots, n$; satisfying to equalities

$$
\text { a) } \lim _{t \rightarrow \infty} \frac{x_{\mu k}}{x_{k k}}=0, \mu \neq k, \quad \text { b) } \lim _{t \rightarrow \infty}\left(\frac{1}{\psi(t)} \frac{x_{k k}^{\prime}}{x_{k k}}-\frac{a_{k}(t)}{\psi(t)}\right)=0
$$

From $b$ ) it follows that for any $\varepsilon>0$ there exists such $T \in I$, that for any $t>T$, $k=1, \ldots, n$; inequalities take place

$$
a_{k}(t)-\varepsilon \psi(t)<\frac{x_{k k}^{\prime}}{x_{k k}}<a_{k}(t)+\varepsilon \psi(t) .
$$

Integrating, we receive

$$
\int_{t_{0}}^{t} a_{k}(\tau) d \tau-\varepsilon \int_{t_{0}}^{t} \psi(\tau) d \tau<\ln \frac{\left|x_{k k}(t)\right|}{\left|x_{k k}\left(t_{0}\right)\right|}<\int_{t_{0}}^{t} a_{k}(\tau) d \tau+\varepsilon \int_{t_{0}}^{t} \psi(\tau) d \tau .
$$

Therefore, inequalities take place

$$
\frac{1}{q(t)} \int_{t_{0}}^{t} a_{k}(\tau) d \tau-\varepsilon<\frac{1}{q(t)} \ln \frac{\left|x_{k k}(t)\right|}{\left|x_{k k}\left(t_{0}\right)\right|}<\frac{1}{q(t)} \int_{t_{0}}^{t} a_{k}(\tau) d \tau+\varepsilon .
$$


From $a$ ) it follows that the $k$-th coordinate of the solution $x_{k}$ is the leader, which implies that the equalities take place

$$
\varlimsup_{t \rightarrow+\infty} \frac{1}{q(t)} \ln \left|x_{k}(t)\right|=\varlimsup_{t \rightarrow+\infty} \frac{1}{q(t)} \int_{t_{0}}^{t} a_{k}(\tau) d \tau=\lambda_{k}\left(A_{d}\right)
$$

Here $\lambda_{1}\left(A_{d}\right), \ldots, \lambda_{n}\left(A_{d}\right)$ are Lyapunov's generalized exponents of the system (0.4), besides they are different. Therefore, the fundamental system of solutions $x_{1}, x_{2}, \ldots, x_{n}$ organizes a normal base of the linearly perturbed system (0.5). Therefore, by the definition

$$
\varlimsup_{t \rightarrow+\infty} \frac{1}{q(t)} \ln \left|x_{k}(t)\right|=\lambda_{k}\left(A_{d}+P\right), \quad k \in\{1, \ldots, n\} ;
$$

are Lyapunov's generalized exponents of the system (0.5) and the equalities take place

$$
\lambda_{i}\left(A_{d}+P\right)=\lambda_{i}\left(A_{d}\right), \quad i=1, \ldots, n ;
$$

Therefore, the linear system (0.4) has Lyapunov's stable generalized exponents. Theorem 1 is proved.

Theorem 0.4. The generalized exponentially separated linear system (0.1) satisfying to the condition (0.2) has Lyapunov's stable generalized exponents.

Proof. By the definition the linear system $(0.1)$ has solutions $x_{1}(t), \ldots, x_{n}(t)$, for which at all $t \geq s \geq t_{0}$ inequalities are fulfilled

$$
\frac{\left\|x_{i-1}(t)\right\|}{\left\|x_{i-1}(s)\right\|} \div \frac{\left\|x_{i}(t)\right\|}{\left\|x_{i}(s)\right\|} \geq B e^{\alpha[q(t)-q(s)]}, \quad i=\overline{2, n}
$$

with some constants $\alpha>0, B \geq 1$.

Hence, it follows that the solutions $x_{1}(t), \ldots, x_{n}(t)$ have various generalized indices, therefore from the property of the generalized indices it follows that they organize a fundamental system of solutions of the linear system (0.1).

Let

$$
x_{i}(t)=\left\|x_{i}(t)\right\| \varphi_{i}(t) \text { where } \varphi_{i}(t)=\frac{x_{i}(t)}{\left\|x_{i}(t)\right\|}, \quad i=\overline{1, n} .
$$

and we present a fundamental matrix of solutions in an aspect

$$
X=\Phi D
$$

where

$$
\Phi(t)=\left[\varphi_{1}, \ldots, \varphi_{n}\right], \quad|\operatorname{det} \Phi|>0, \quad D=\operatorname{diag}\left(\left\|x_{1}\right\|,\left\|x_{2} \mid, \ldots,\right\| x_{n} \|\right)
$$

The equalities take place

$$
\frac{1}{2\left\|x_{i}(t)\right\|^{2}} \frac{d\left\|x_{i}(t)\right\|^{2}}{d t}=\frac{\left(A(t) x_{i}(t), x_{i}(t)\right)}{\left\|x_{i}(t)\right\|^{2}}=\left(A(t) \varphi_{i}(t), \varphi_{i}(t)\right)=a_{i}(t)
$$

where $a_{i}(t), i=1, \ldots, n$; continuous functions at $t \geq t_{0}$. 
Hence integrating, for any $t \geq s \geq t_{0}$ we receive

$$
\left\|x_{i}(t)\right\|=\left\|x_{i}(s)\right\| \exp \left(\int_{s}^{t} a_{i}(\tau) d \tau\right), \quad i=1, \ldots, n ;
$$

From (0.8), (0.10) it follows that

$$
\exp \left(\int_{s}^{t}\left(a_{i-1}(\tau)-a_{i}(\tau)\right) d \tau\right) \geq B \exp [\alpha(q(t)-q(s))], \quad i=2, \ldots, n
$$

This implies that the inequalities are fulfilled

$$
a_{i-1}(t)-a_{i}(t) \geq \alpha \psi(t), \quad \alpha>0, t \in I, i \in\{2, \ldots, n\},
$$

Let us carry out transformation in the system (0.1) taking as a matrix of transformation the matrix $\Phi(t)$

$$
x=\Phi y
$$

Then we receive the linear system

$$
\dot{y}=D^{*} y
$$

where

$$
D^{*}=\Phi^{-1} A \Phi-\Phi^{-1} \dot{\Phi}
$$

Note that in the transformation (0.12), matrixes $\Phi(t), \Phi^{-1}(t)$ are continuous limited, and the matrix $\dot{\Phi}(t)$ is continuous and $\|\dot{\Phi}(t)\| \leq K \psi(t)$. Therefore, $(0.12)$ is the generalized Lyapunov's transformation.

To the fundamental matrix $X$ linear to the system (0.1) there corresponds the fundamental matrix $Y$ of the linear system (0.13) and from the equality

$$
X=\Phi Y
$$

it follows that

$$
Y=\Phi^{-1} X
$$

Substituting (0.9) in (0.14) we have

$$
Y=\Phi^{-1} \Phi D=D=\operatorname{diag}\left(\left\|x_{1}(t)\right\|, \ldots,\left\|x_{n}(t)\right\|\right)
$$

or

$$
Y=\operatorname{diag}\left(\left\|x_{1}\left(t_{0}\right)\right\| e^{\int_{0}^{t} a_{1} d s}, \ldots,\left\|x_{n}\left(t_{0}\right)\right\| e^{\int_{t_{0}}^{t} a_{n} d s}\right)
$$

The equation has such a fundamental system of solutions

$$
\dot{y}=D y
$$

where

$$
D=\operatorname{diag}\left(a_{1}, a_{2}, \ldots, a_{n}\right)
$$


Therefore, owing to uniqueness the equality takes place

$$
D^{*}=D
$$

Thus, the generalized exponentially separated linear system (0.1) satisfying to the condition ( 0.2 ) by application of Lyapunov's generalized transformation is reduced to a diagonal system satisfying to the condition (0.11). As Lyapunov's generalized transformation keeps stability, from theorem 1 it follows that Lyapunov's generalized exponents of the linear system (0.1) are stable.

Theorem 2 is proved.

Example 0.5. Let us consider the linear system

$$
\left\{\begin{array}{l}
\dot{x}=-\frac{1}{4 \sqrt{t}} x+\frac{\sin t}{t+1} y \\
\dot{y}=\frac{\cos t}{t+2014} x-\frac{1}{2 \sqrt{t}} y
\end{array},(t>1)\right.
$$

Note that the linear system from diagonal coefficients of this system has Lyapunov's generalized exponents $\lambda_{1}(q)=-\frac{1}{2}, \lambda_{2}(q)=-1$, where $q(t)=\sqrt{t}$ and the diagonal system is generalized exponentially separated, and the approval of theorem 2 is fulfilled. Therefore, this system has the same Lyapunov's generalized exponents. This implies that the system is stable according to Lyapunov. 


\section{REFERENCES}

[1] Aldibekov T.M. The Analog of Lyapunov's Theorem on Stability at the First Approximation // Differential Equations. - 2006. - V.42, ${ }^{1} 6$. - p.p. 859-860.

[2] Aldibekov T.M. On Stability at the First Approxmation // Journal "Modern Problems of Science and Education" Moscow. - 2008. - p. 133.

[3] Aldibekov T.M. Lyapunov's Generalized Exponents. - Almaty., 2011. - 254 p.

[4] Aldibekov T.M., Aldazharova M.M. On the Stability in the First Approximation in Critical Cases of Lyapunov Characteristic Exponents // Differential Equations. - 2013. - Vol. 49, No. 6. - p. 2013.

[5] Anosov D.V. Geodesic Flows on the Closed Riemannian Varieties of Negative Curvature. Tr. Math. Inst. after Steklov V.A., V. XC. M., "Nauka", 1967.

[6] Bylov B.F. On Linear Equation System Reduction to a Diagonal Aspect // Mathematical Collection. - 1965. - V. $67,{ }^{1} 3$. - p.p. $338-344$.

[7] Vinograd R.E. DAN USSR. - 1958. - V. $119,{ }^{1} 4$. - p.p. $633-635$.

[8] Lillo J.C. Acta Math., 103, 1960, 123-128.

[9] Millionshchikov V.M. Systems with Integral Separation are Dense Everywhere in the Set of Linear Systems of Differential Equations // Differential Equations. - 1969. - V.5, ${ }^{1} 7 .-$ p.p. $1167-1170$

[10] Millionshchikov V.M. On exponents of Exponential Separation // Mathematical Collection. - 1984. - V.124 (166). ${ }^{1} 4$ - p.p. 451-485.

[11] Nemytskii V.V., Stepanov V.V. Qualitative Theory of Differential Equations. - M - L.: Gostekhizdat, 1949. - $551 \mathrm{p}$.

[12] Perron O. Uber lineare Differentialgleichungen, bei denen die unabhangige Variable reel ist. J. Reine und angew. // Math., - 1931. - B.142. - p.p. 254-270.

Current address: Al-Farabi Kazakh National University, 